\title{
Silicon micromachined ultrasonic scalpel for the dissection and coagulation of tissue
}

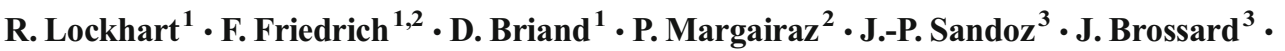 \\ H. Keppner ${ }^{3}$ - W. Olson ${ }^{4}$ - T. Dietz ${ }^{4}$ - Y. Tardy ${ }^{2}$ - H. Meyer ${ }^{6}$ • P. Stadelmann ${ }^{6}$. \\ C. Robert $^{6}$ - A. Boegli ${ }^{6}$ • P.-A. Farine ${ }^{6}$ - N. F. de Rooij $^{1} \cdot$ J. Burger $^{2,5,7}$
}

Published online: 8 July 2015

(C) Springer Science+Business Media New York 2015

\begin{abstract}
This work presents a planar, longitudinal mode ultrasonic scalpel microfabricated from monocrystalline silicon wafers. Silicon was selected as the material for the ultrasonic horn due to its high speed of sound and thermal conductivity as well as its low density compared to commonly used titanium based alloys. Combined with a relatively high Young's modulus, a lighter, more efficient design for the ultrasonic scalpel can be implemented which, due to silicon batch manufacturing, can be fabricated at a lower cost. Transverse displacement of the piezoelectric actuators is coupled into the planar silicon structure and amplified by its horn-like geometry. Using finite element modeling and experimental displacement and velocity data as well as cutting tests, key design parameters have been identified that directly influence the power efficiency and robustness of the device as well as its ease of controllability when driven in resonance.
\end{abstract}

J. Burger

jburger@campus.unibe.ch

1 Ecole Polytechnique Fédérale de Lausanne (EPFL), Institute of Microengineering (IMT), Sensors, Actuators and Microsystems Laboratory (SAMLAB), Neuchâtel, Switzerland

2 Medos International, A Johnson \& Johnson Company, Le Locle, Switzerland

3 Haute Ecole Arc Ingénierie, Neuchâtel, Switzerland

4 Ethicon Endo-Surgery, Cincinnati, OH, USA

5 Institute for Surgical Technology and Biomechanics, University of Bern, Bern, Switzerland

6 Ecole Polytechnique Fédérale de Lausanne (EPFL), Electronics and Signal Processing Lab, Neuchâtel, Switzerland

7 Zurich University of Applied Sciences, Institute of Mechatronic Systems, Technikumstrasse 5, CH-8401 Winterthur, Switzerland
Designs in which the full- and half-wave transverse modes of the transducer are matched or not matched to the natural frequencies of the piezoelectric actuators have been evaluated. The performance of the Si micromachined scalpels has been found to be comparable to existing commercial titanium based ultrasonic scalpels used in surgical operations for efficient dissection of tissue as well as coaptation and coagulation of tissue for hemostasis. Tip displacements (peak-to-peak) of the scalpels in the range of $10-50 \mu \mathrm{m}$ with velocities ranging from 4 to $11 \mathrm{~m} / \mathrm{s}$ have been achieved. The frequency of operation is in the range of $50-100 \mathrm{kHz}$ depending on the transverse operating mode and the length of the scalpel. The cutting ability of the micromachined scalpels has been successfully demonstrated on chicken tissue.

Keywords Ultrasonic cutter · Ultrasonic scalpel · Silicon · Micromachined acoustic horn $\cdot$ High power acoustic transducer · Piezoelectric

\section{Introduction}

Since its first application for phacoemulsification by Kelman in 1967 (Kelman 1967), ultrasonic devices for surgical applications have been widely recognized to be efficient and safe for the cutting, ablation or fragmentation, and removal of biological tissue. Initially conceived for surgical interventions in ophthalmology and laparoscopic abdominal surgery, ultrasonic surgery techniques are nowadays widely spread in a large number of different medical disciplines due to their numerous advantages (O’Daly et al. 2008, Pavlíková et al. 2011).

Ultrasonic scalpels are precise and effective cutting tools that minimize thermal tissue damage. In addition to dissection 
of tissue, ultrasonic scalpels allow the surgeon to simultaneously perform coaptation and coagulation for hemostasis (Melck and Wiseman 2010).

In general, it is assumed that ultrasonic cutting tools disperse less energy into neighboring tissue and cause less proximal lateral damage and tissue necrosis compared to current high-frequency technologies (Metternich et al. 2003, Amaral 1994, Boddy et al. 1987).

The basic mechanism of ultrasonic scalpels is the fast longitudinal oscillating displacement of the instrument tip that provides a cutting effect by generating shear force and a static pressure in the cells above the mechanical yield of the tissue (Ebina et al. 2007). Furthermore, cavitation occurs when the ultrasonic energy is transmitted to the tissue, causing an oscillating acoustic pressure field which results in a oscillating volume change of the tissue and cell fluid. Thus, during the expansion phase, the pressure of the cellular or tissue liquid can fall below its vapor pressure and vapor bubbles are generated. With the increasing pressure during the compression phase, these bubbles collapse by generating shock waves and jet-like ejections into the fluid finally leading to the dissection of the tissue. Depending mainly on the nature of the tissue and secondly on its water content, this effect is highly tissue-selective. According to the results of Cimino et al., the tissue strength which is directly related to the type, quantity and organization of collagen is the most important physiological factor for the fragmentation performance of ultrasonic surgery (Cimino and Bond 1996, Bond and Cimino 1996a).

The reciprocating excursion of the blade part is also responsible for the thermal impact, since it creates heat due to the friction between blade and tissue which, depending on the applied time, leads to coaptation or coagulation of the cells. By disrupting the hydrogen bonds, causing collagen molecules to collapse and adhere to one another the coaptation, or adherence of tissue, is achieved at low temperatures $\left(<80^{\circ} \mathrm{C}\right)$. If the ultrasonic energy is applied for a few seconds longer, the proteins will be denatured and coagulate due to higher temperatures $\left(<150^{\circ} \mathrm{C}\right)$. Both, coaptation and coagulation can be used for the closing of vascular structures and hemostasis whereas the physical phenomenon of cavitation is only used to cut.

On the technical side, the amplitude of the instrument's tip displacement and its velocity have been identified by several studies (Bond and Cimino 1996b, Lal 1996, Vaitekunas et al. 2001) to be the most influencing factors on the cutting rate of an ultrasonic instrument. For this reason, optimization of the design with respect to its tip displacement plays a key role in the development of a silicon micromachined ultrasonic scalpel.

Ultrasonic cutting instruments are widely used in various fields of surgery including: peripheral vascular surgery (Cikirikcioglu et al. 2001); colorectal surgery (Armstrong et al. 2001); bariatric surgery (Stoff et al. 2007); breast surgery
(Lumachi et al. 2004); general and visceral surgery (Takao et al. 2000); ENT surgery (Walker and Syed 2001); gynecologic surgery (Kauko 1998); thoracic surgery (Watanabe et al. 1998); plastic surgery (Albert et al. 2012, Burdette et al. 2011); urologic surgery (Sasagawa et al. 2000); CMF surgery (Sherman and Davies 2000); and, cardiac and cardiovascular surgery (Lamm et al. 2000, Brazio et al. 2008). Most recent advances in the use of ultrasonic scalpels include robotassisted computer enhanced closed-chest coronary surgery (Kiaii et al. 2000).

However, due to their current size, weight and cost, the use of ultrasonic scalpels has been limited to certain application fields. Here, we present the design of an ultrasonic scalpel, based on a concept for silicon ultrasonic tools developed by Lal et al. (Lal and White 1996) which consists of a micromachined silicon horn that resonates longitudinally due to two PZT actuators bonded to either face of the horn and driven in the transverse mode (d31) as shown in Fig. 1. Sadiq et al. and Kuang et al. have developed a planar cutting tool using Ti and stainless steel for the ultrasonic waveguide as well as piezoelectric actuators in the $\mathrm{d}_{31}$-mode for longitudinal excitation of the waveguide at its resonant frequency (Kuang et al. 2013; Sadiq et al. 2011). However, they observe low displacement amplitudes of 5-6 $\mu \mathrm{m}$ using a driving amplitude of $50 \mathrm{Vp}-\mathrm{p}$ as well as nonlinearities in the form of frequency shifts when driving the ultrasonic waveguide in resonance. Nagoya et al. report of a micro ultrasonic scalpel composed of a Ti waveguide with thickness $0.1 \mathrm{~mm}$, width $1 /$ $0.1 \mathrm{~mm}$ at the low / high amplitude side and length $9,8 \mathrm{~mm}$ (Nagoya et al. 2003). Their design reached a vibration velocity in longitudinal direction of up to $2.2 \mathrm{~m} / \mathrm{s}$ at a driving voltage of 30.0 Vpeak-peak.

The ultrasonic scalpel is light, powerful, and more cost-effective than the currently used ultrasonic tools. Silicon has a higher speed of sound and thermal conductivity as well as a lower density than the titanium based alloys that are currently used. The relatively high Young's modulus of Si enables a lighter, more efficient design, but perhaps most importantly, the production cost is vastly reduced due to batch processing by microfabrication technologies such as anisotropic etching. The major disadvantage of using silicon as material for the ultrasconic waveguide of surgical instruments is its brittleness which could result in cracking at high stress. These failures are due to always existing cracks and defects on surfaces. A possibility to avoid the cracking would include the coating of silicon with a hard material like e.g., $\mathrm{Si}_{3} \mathrm{~N}_{4}$ or $\mathrm{SiC}$.

The focus of this work was to improve the existing concept and optimize the device for use in high power applications by identifying key design parameters and important performance indicators to increase the robustness and reliability of the Si scalpels as well as their ability to function under mechanical loads. 
PZT Actuators

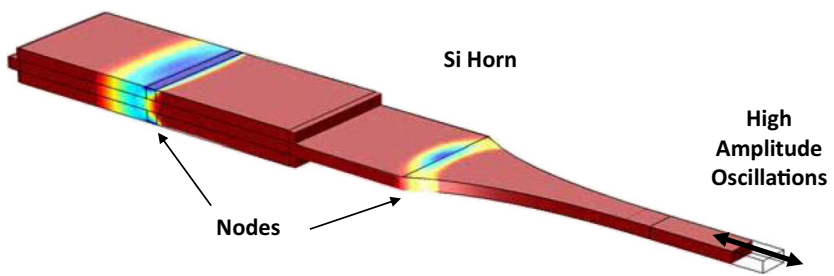

Fig. 1 Schematic of the Si micromachined Ultrasonic scalpel

\section{Materials and methods}

\subsection{Transducer description}

The silicon micromachined ultrasonic scalpel for the dissection and/or coagulation of patient tissue consists of an ultrasonic transducer assembly including a longitudinally elongated, planar micromachined silicon waveguide. The silicon waveguide resonates longitudinally due to two PZT actuators bonded to either face of the waveguide and driven in the transverse piezoelectric mode (d31) as shown in Fig. 1.

\subsection{Material selection}

Silicon was chosen as the material for the planar waveguide as the mechanical and thermal properties of silicon qualify it as an ideal material for high power applications in MEMS, and hence, ultrasonic scalpels. First, silicon has a linear elastic behavior up to a high yield strength of approximately $7 \mathrm{GPa}$ (Petersen 1982), compared to $0.9 \mathrm{GPa}$ for the titanium alloy $\mathrm{C}-120 \mathrm{AV}$ which has been traditionally used for ultrasonic scalpels which equates to the ability to endure high mechanical stresses. Second, silicon has a high thermal conductivity which permits efficient transport of frictional heat produced along the blade to avoid exceedingly high temperatures and the risk of tissue damage.

A 'hard' piezoelectric ceramic, PZT-8 (PIC 181 from PI Ceramic $\mathrm{GmbH}$ ), was chosen for the actuators since it is able to support high electrical and mechanical stress making it ideal for high-power applications. PIC 181 is a modified lead zirconate titanate piezoelectric ceramic that has a high Curie temperature $\left(\mathrm{T}_{\text {Curie }}=330^{\circ} \mathrm{C}\right)$, a high mechanical quality factor $(Q=2000)$ and a low dielectric loss $(\tan \delta=0.003)$. It also exhibits good temperature and time stability of its dielectric and mechanical constants making it a good choice for resonant-mode ultrasonic applications.

The components are bonded together with DELOMonopox ${ }^{\mathrm{TM}} \mathrm{AD} 066$ which is thermally cured between 120 and $150^{\circ} \mathrm{C}$ (well below the curie temperature of PIC 181) in a relatively short time (20 min.). It possesses a high tensile strength of $50 \mathrm{MPa}$ and a shore hardness of 83D.

\subsection{Silicon horn fabrication}

Two methods were used to fabricate the silicon waveguide. In the first method, the silicon waveguide was laser cut from a $1 \mathrm{~mm}$ thick monocrystalline (100)-Si-wafer with an Nd:YAG laser using frequency tripling $(\lambda=$ $355 \mathrm{~nm}$ ), a spot size of $20 \mu \mathrm{m}$ and a maximum output power of $2 \mathrm{~W}$. This process is ideal for prototyping new designs; however, the laser ablation procedure introduces micro-cracks along the surface of the silicon horn. The extremely high brittleness of silicon in combination with the presence of defects can lead to abrupt material failure below the theoretical yield strength of silicon. In order to avoid the propagation of micro-cracks from the surface to the interior of the silicon horn when it is excited at ultrasonic frequencies, it is important to achieve a very high surface quality by applying additional surface treatments (Ericson and Schweitz 1990) or avoiding mechanical cutting techniques (Funke et al. 2009). Therefore, in order to improve the surface quality of the $\mathrm{Si}$ waveguides and reduce the surface defects induced by laser-cutting, a $1.95 \mu \mathrm{m}$ high quality thermal oxide layer was grown on the surface of the $\mathrm{Si}$ waveguide using a wet oxygen oxidation at $1200{ }^{\circ} \mathrm{C}$. The oxidation time for a $1.95 \mu \mathrm{m}$ oxide layer has been calculated using the Deal-Grove model and was $5 \mathrm{~h} 20 \mathrm{~min}$ for (100) silicon.

The addition of the thermal oxide layer led to a significant decrease in the number of device failures at high displacement amplitudes.

An anisotropic wet etchant (potassium hydroxide $\mathrm{KOH}$ ) was also used as an alternative approach to fabricate the silicon waveguides while at the same time exposing the [111] planes along the tip of the scalpel to produce a sharp blade as shown in Fig. 2. A final oxidation of the surface was also used with this patterning method to improve the reliability of the scalpels. The extension and contraction axis of the scalpel was chosen to lie along the $<110>$ crystallographic direction of the silicon wafer resulting in a Young's modulus of $169 \mathrm{GPa}$ for the waveguide. The chosen configuration permits a sharp blade to be formed along the length of the tip using standard $\mathrm{KOH}$ processing of a (100) wafer.

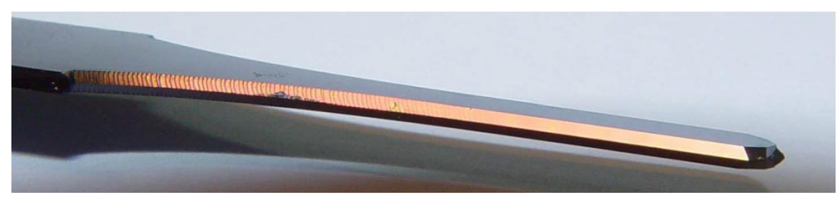

Fig. 2 Anisotropically etched Si scalpel blade 
The basic assembly procedure for a micromachined scalpel is shown in Fig. 3. The piezoelectric PZT actuators are diced, and cleaned in acetone and isopropanol. In order to provide electrical contacts, the PZT plates are cut $2.5 \mathrm{~mm}$ wider than the width of the Si-waveguide so that the PZT extends over the edge of the $\mathrm{Si}$ and provides access to solder the contact wires.
Gluing of the PZT actuators is done by manual application or spin coating of a thin layer of a high-strength, heat-resistant epoxy adhesive (DELOMonopox ${ }^{\mathrm{TM}} \mathrm{AD} 066$ ) to one surface of each of the PZT pieces. One of the PZT actuators is pressed onto the surface of the $\mathrm{Si}$ waveguide which is placed in an oven for $5 \mathrm{~min}$ at $140{ }^{\circ} \mathrm{C}$ to pre-cure the glue. The second PZT
Fig. 3 Schema of the fabrication process

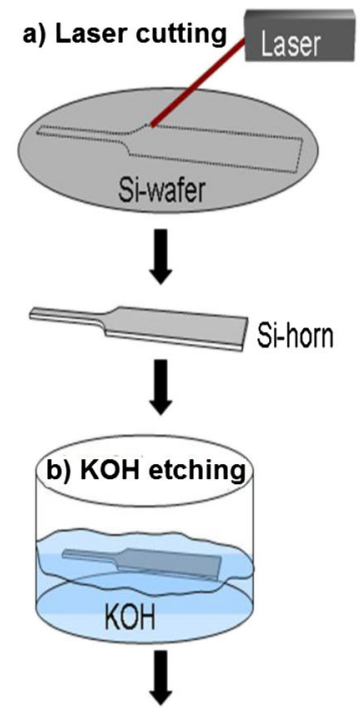

c) Drying

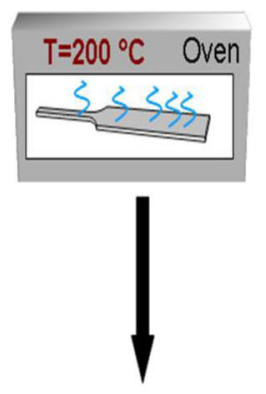

d) Laser cutting

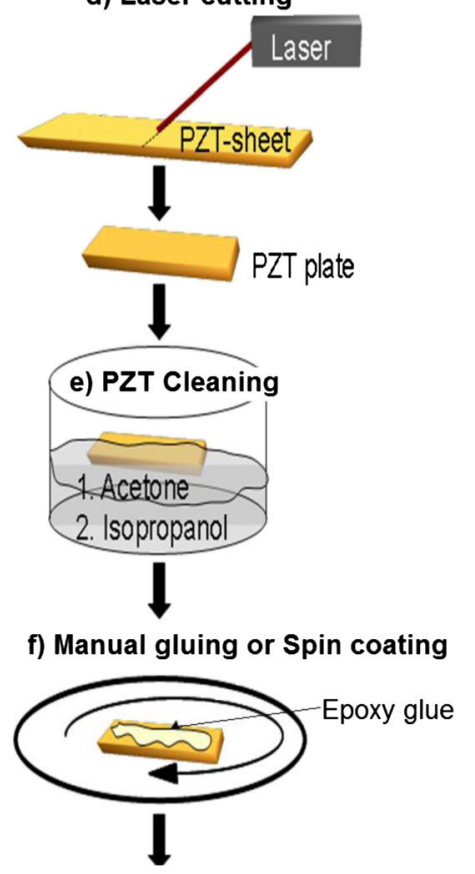

g) UV-activation

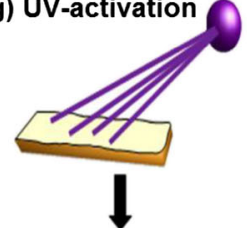

h) manual assembly+removal of glue residua from contacting surfaces

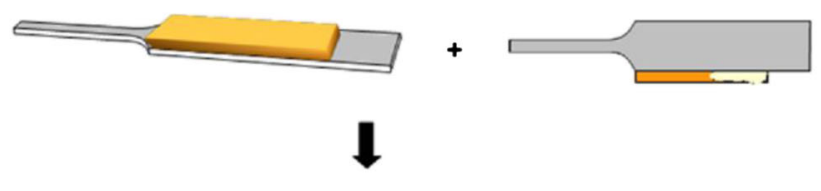

i) curing
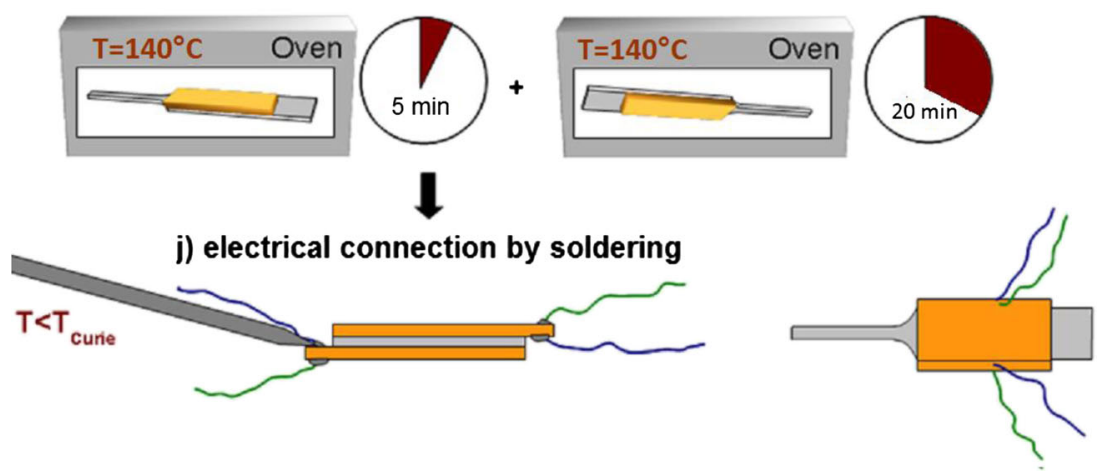


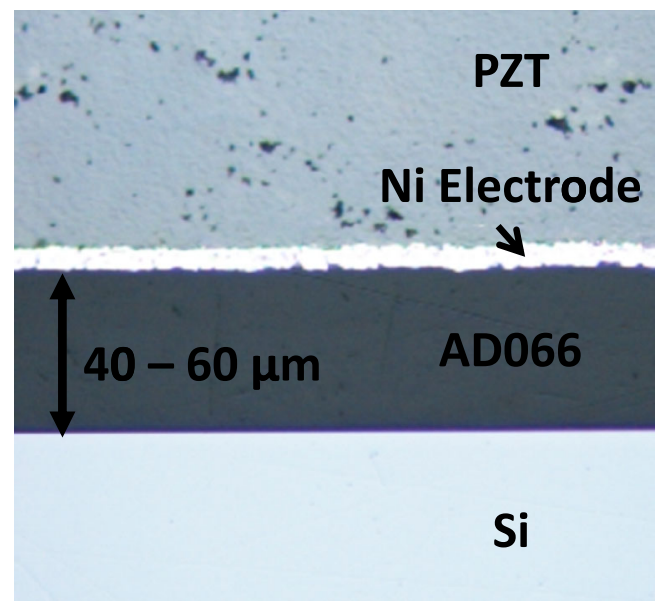

Fig. 4 Sectional view of an assembled scalpel with an approx. 40-60 $\mu \mathrm{m}$ thick bonding layer of the epoxy adhesive AD066

actuator is then placed on the opposite surface of the $\mathrm{Si}$ and the fully assembled waveguide is placed in the oven to complete the curing at $140{ }^{\circ} \mathrm{C}$ for $20 \mathrm{~min}$. This process results in bonding layer thicknesses of $\sim 40-60 \mu \mathrm{m}$ on either side of the $\mathrm{Si}$ waveguide (Fig. 4).

The piezoelectric plates can be asymmetrically assembled to provide access to the under electrode of the PZT plate. Alternatively, a small amount of conductive epoxy along the back end of the PZT plate can be used to make the electrical connection to the underside of the PZT plates. The latter option provides a cleaner, more compact solution.

\subsection{Characterization}

Characterization of the scalpels was used to determine the frequency of operation for each mode, the displacement of the scalpel tip as a function of drive voltage and current, the ability of the scalpel to function under loading conditions, the nodal positions, and the temperature stability of the scalpels.

Characterization of the devices with respect to performance and efficiency was realized by first performing an impedance analysis to determine the electrical equivalent circuit and to define the impedance curve of the device. These results are used to calculate the effective electromechanical coupling factor, $\mathrm{k}_{\mathrm{eff}}$ of the assembled ultrasonic scalpel. Next, the scalpels were excited by sweeping over their resonance frequency at various voltages while measuring the tip displacement using a laser Doppler vibrometer. The current passing through the piezoelectric plates and the phase between the input voltage signal and the measured signal was also recorded. An electrical schematic of the characterization setup is shown in Fig. 5.

\subsubsection{Impedance analysis}

An HP4294A impedance analyzer was used to measure the impedance and phase of the microscalpels as a function of frequency. This analysis was used to visualize the operational modes of the scalpels, both longitudinal and transverse (bending). The scalpels were scanned in the expected zone of operation between 50 and $120 \mathrm{kHz}$ (Fig. 6). The frequency range was then reduced to focus on each of the peaks found in the initial scan.

Impedance analysis was also used to confirm improper operation of scalpels damaged during cutting and highamplitude testing. In some cases, breakage of the silicon horn was clearly observable; however, in many cases, scalpels that failed during testing had no observable damage other than diminished performance. These scalpels were found to produce a double resonance peak around the natural frequency of the actuators. The double peak is thought to be caused by damage to the extremities of the piezoelectric actuator on
Fig. 5 Schematic of the circuit used to characterize current and phase of the scalpels as a function of applied frequency while measuring the displacement of the tip with the laser Doppler Vibrometer (LDV)

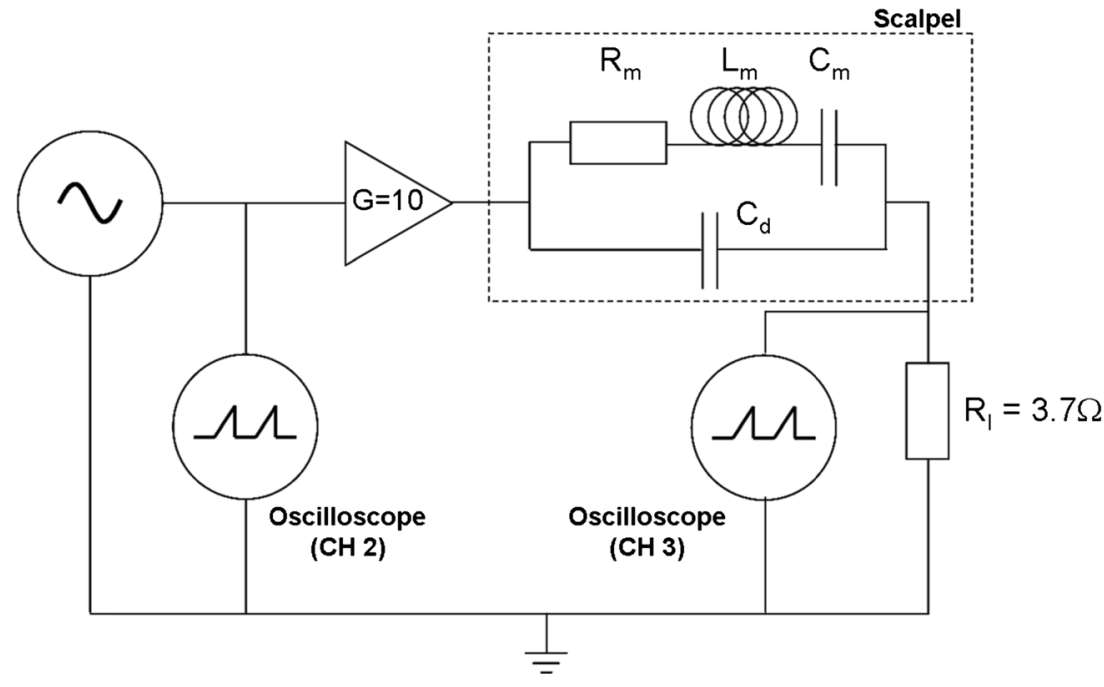


Fig. 6 Impedance and phase spectrum of a Si scalpel with design $\mathrm{B}$ showing the matched $\mathrm{PZT} /$ system half-wave $(\lambda / 2)$ mode at $57.7 \mathrm{kHz}$ and the unmatched PZT/system full-wave (入) mode at $74.8 \mathrm{kHz}$

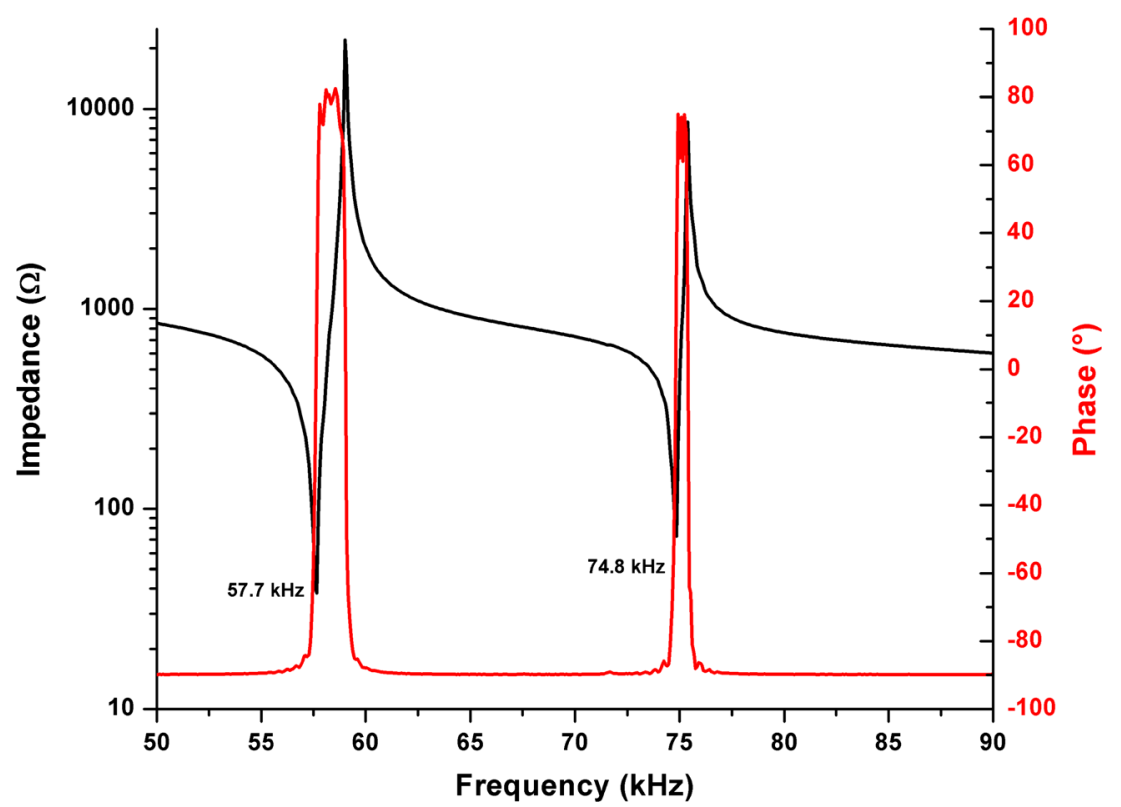

one side of the assembly which behaves differently than the piezoelectric actuator on the opposite side.

\subsubsection{Nodal determination}

The nodal positions along the scalpel (the points at which no longitudinal contraction or extension takes place) were determined using the measured impedance of the scalpels while being driven in resonance. By varying the clamping position to minimize the impedance, the nodal positions could be easily located.

\subsubsection{Transducer displacement and velocity}

A laser Doppler vibrometer (LDV) was used to measure the velocity, and thus the longitudinal and transverse displacements of both the silicon waveguide and the PZT actuators at different points along the length of the transducer as a function of driving frequency. The scalpel was connected as shown in Fig. 5 in order to also measure the current and phase of the scalpel under test. The signal from a function generator (HP4294A) passes through a high-power amplifier with a gain of $10\left(\mathrm{I}_{\max }=1.2 \mathrm{~A}\right)$. At the output of the amplifier the positive lead was connected directly to the one of the scalpel leads while the other (ground) was first passed through a $3.7 \Omega\left(\mathrm{P}_{\max }=10 \mathrm{~W}\right)$ resistor. The voltage across the resistor was measured on the oscilloscope and used to determine the current passing through the scalpel $\left(I=V_{i} / R_{i}\right)$. The phase between the measured current and the voltage signal from the function generator was also measured on the oscilloscope.

Longitudinal displacement measurements were performed using the LDV to determine the displacement amplitude of the scalpel tip as well as the ends of PZT actuators. The laser from the LDV head was focused on the point to be measured (either the tip of the scalpel or the facet of the PZT). Computer control through a GPIB connection was used to automatically vary the amplitude and frequency supplied by the function generator and record the measured displacement, current, and phase.

\subsubsection{Operation under loading conditions}

Tests were performed with and without a static load placed on the tip of the scalpel during operation. Application of the load reduces the amplitude of the oscillations as expected requiring higher voltages to be applied in order to reach amplitudes equivalent to the load-free test case. Scalpels that exhibit a higher gain are more easily damped and therefore require a greater relative increase in the applied voltage to maintain a constant displacement. Reducing the gain of the scalpel decreases the variation in the applied voltage that is required over a range of loading conditions (see also Fig. 10). As a result, this leads to scalpels that are able to adapt to the varying loading conditions found in cutting applications, reducing failures caused by excess strain in the horn and actuators when the applied voltage is not adapted to fit the changing loading conditions on the scalpel.

\section{Design and modelling}

\subsection{Transducer geometry}

The total energy of the waveguide is based on the presence of inertial and elastic forces, whose amount depends amongst others on the cross-sectional area and more particularly on 
Table 1 Profiles w(x) of different ultrasonic waveguide designs, definitions and properties ((Wang et al. 2011), Lal 1996). A modified stepped waveguide has been chosen for the Si micromachined scalpel due to its reduced stress concentration and good displacement amplification. For the definitions of dimensions see Fig. 7

\begin{tabular}{|c|c|}
\hline Caternary & $\begin{aligned} w(x) & =w_{S i} \cdot\left(\cosh \left(a \cdot\left(L_{S i}-x\right)\right)\right)^{2} ; 0<x<L_{S i} \\
\alpha & =\frac{1}{L_{2}} \cdot \cosh ^{-1}\left(\sqrt{\frac{w_{S i}}{w_{\text {tip }}}}\right)\end{aligned}$ \\
\hline Stepped & $w(x)=\left\{\begin{array}{c}w_{S i} ; x<\frac{L_{S i}}{2} \\
w_{t i p} ; x>\frac{L_{S i}}{2}\end{array}\right.$ \\
\hline Modified stepped & $\begin{aligned} w(x) & =\left\{\begin{array}{c}w_{S i} ; x<L_{1} \\
w_{S i} \cdot\left(\cosh \left(a\left(\left(L_{1}+L_{2}\right)-x\right)\right)\right)^{2} ; L_{1}<x<L_{1}+L_{2} \\
w_{t i p} ; x>L_{1}+L_{2}\end{array}\right. \\
\alpha & =\frac{1}{L_{2}} \cdot \cosh ^{-1}\left(\sqrt{\frac{w_{S i}}{w_{\text {tip }}}}\right)\end{aligned}$ \\
\hline
\end{tabular}

the waveguide width. The design of ultrasonic waveguides presents a trade-off between amplification and mechanical reliability which has been studied extensively by many groups (Lal 1996, Wang et al. 2011, Eisner 1964). We combine the advantages of a stepped waveguide and a catenary waveguide into a hybrid design, called the modified stepped waveguide (Table 1). Compared to a fully catenary horn, it offers high amplification and a rectangular surface for the rectangular PZT plates.

In this study, we focus on two geometrical transducer designs both based on the modified stepped profile. The first, designs $\mathrm{A} 1$ and $\mathrm{A} 2$, have a high aspect ratio between the narrow, high amplitude tip and the wide, low amplitude base; thus, it generates a larger displacement amplification. The aspect ratio in designs $\mathrm{A} 1$ and $\mathrm{A} 2$ is $A_{\text {low }}$ $A_{\text {high }}=8$, where $A_{\text {low }}$ is the cross sectional area of the low amplitude section and $A_{\text {high }}$ is the cross sectional area of the high amplitude section. The thickness is the same all along the planar transducer; therefore, the ratio of cross sectional areas only depends on the width of each section. Thus, in designs A1 and A2, the width of the low amplitude section is $20 \mathrm{~mm}$ and the width of the high amplitude section is $2.5 \mathrm{~mm}$. Design B uses a more subtle transition, passing from a low amplitude width of $10 \mathrm{~mm}$ to the same high amplitude width of $2.5 \mathrm{~mm}$ providing a cross sectional area ratio of four. The ratio between these two sections is proportional to the geometric amplification of the transducer; hence, designs $\mathrm{A} 1$ and $\mathrm{A} 2$ are expected to have a larger geometric amplification than design B.

The first fundamental, purely longitudinal mode of the resonator is the half-wave $(\lambda / 2)$ mode in which the transducer has a single nodal position near its center and two anti-nodes at its extremities. The second fundamental, purely longitudinal mode, the full-wave $(\lambda)$ mode, has two nodal positions and three anti-nodes - two at the extremities and one along the length of the transducer. The locations of the nodal positions (and the position of the anti-node in the $\lambda$ mode) depend on the geometry of the transducer - the width ratio and transition length of the transducer and the size of the piezoelectric actuators. The transducers can be designed such that the natural frequencies of the Si horn are matched to the natural frequencies of the piezoelectric actuators (for a chosen mode of operation) or they can be designed to be not matched. In the matched case, the piezoelectric actuators experience large displacements (as a result of self-resonance) and therefore suffer from hysteresis making it difficult for control electronics to pin-point the operational frequency; however, since resonance of the structure is driven by resonance of the piezoelectric actuator, the transducers are not easily damped and are therefore able to efficiently drive power to an applied load. On the other hand, transducers designed such that the resonance frequency of the piezoelectric actuators is not matched to the resonance frequency of the structure are easier to control, but are also more easily damped by an applied load. 


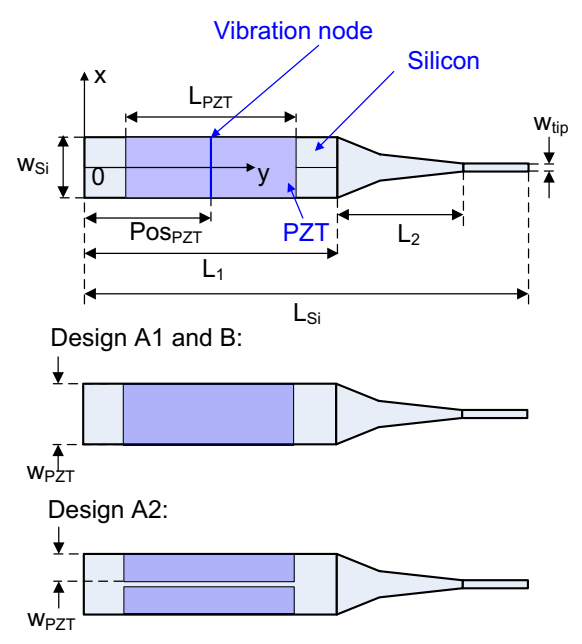

\begin{tabular}{|l|l|l|l|}
\hline Dimension & Design A1 & Design A2 & Design B \\
\hline Note & $\begin{array}{l}\text { Matched } \lambda / 2, \\
\text { Unmatched } \lambda\end{array}$ & $\begin{array}{l}\text { Matched } \lambda / 2, \\
\text { Unmatched } \lambda\end{array}$ & $\begin{array}{l}\text { Matched } \lambda / 2, \\
\text { Unmatched } \lambda\end{array}$ \\
\hline Geometric Gain & 8 & 8 & 4 \\
\hline WSi $[\mathrm{mm}]$ & 20 & 20 & 10 \\
\hline $\mathrm{W}_{\text {tip }}[\mathrm{mm}]$ & 2.5 & 2.5 & 2.5 \\
\hline WPZT $_{\text {P }}[\mathrm{mm}]$ & 22.5 & 9 & 10 \\
\hline L $_{1}[\mathrm{~mm}]$ & 50 & 50 & 40 \\
\hline $\mathrm{L}_{2}[\mathrm{~mm}]$ & 20 & 20 & 20 \\
\hline L $_{\mathrm{Si}}[\mathrm{mm}]$ & 80 & 80 & 70 \\
\hline LPZT $[\mathrm{mm}]$ & 30 & 17.2 & 30 \\
\hline PoS & 31.4 & 22.7 & 15 \\
\hline tPZT $_{\text {PZT }}[\mathrm{mm}]$ & 2 & 1 & 2 \\
\hline
\end{tabular}

Fig. 7 Dimensions of scalpel designs A1, A2, and B

\subsection{Finite element modeling}

3D finite element models of each component (i.e., the PZT plates and the Si horn) in addition to an assembly of the complete scalpel were developed in COMSOL. The models of the individual components were used to determine the resonant modes of the plates and to dimension them appropriately as to eliminate any spurious modes in the frequency range of interest. Actuators with length-to-width ratios equal to approximately 1 were found to exhibit a number of spurious modes that were detrimental to the operation of the scalpel. Therefore, as a design guideline, the length-to-width ratio $(1 / \mathrm{w})$ was set to be greater than 1.5. In designs requiring shorter actuators, the actuators were divided into two along the direction of the width in order to satisfy the minimum length-to-width ratio since the width of the actuator is governed by the width of the silicon waveguide.

In the simulations, an $\mathrm{AC}$ voltage was applied across the piezoelectric actuators and the longitudinal displacement of the scalpel tip was monitored as a function of the voltage amplitude and frequency. An electric circuit was also implemented in the COMSOL model in order to simulate the current flowing through the piezoelectric actuators for each applied voltage. The phase difference between the simulated current and the applied voltage was used to calculate the phase margin of the device. A wide phase margin is important for the control electronics as it is used to pinpoint and track the resonance frequency of the scalpel. In general, designs with a high peak phase angle $\left(\varphi>60^{\circ}\right)$ and a wide phase angle margin (the frequency separation between zero phase crossings) exhibit good controllability. The cutting ability of the scalpel can be estimated from the variation of these values under loading conditions. A slight decrease in the peak phase angle, and the tip displacement, and a small increase in impedance are desirable for adequate cutting performance of the scalpels.

Simulations in which a $1 \mathrm{~N}$ loading force was applied to the tip of the scalpel were used to evaluate the performance of the scalpels under loading conditions. The effect of the load on the tip displacement and phase angle of the scalpel indicates it effectiveness in cutting applications. The force of $1 \mathrm{~N}$ has been selected based on research done by Vaitekunas et al. who

Table 2 Comparison of the properties and the performance of the three scalpel designs A1, A2 and B in the matched and unmatched half- and fullwave modes

\begin{tabular}{|c|c|c|c|c|c|c|}
\hline \multirow{2}{*}{$\begin{array}{l}\text { Resonance mode } \\
\text { Operation mode }\end{array}$} & \multicolumn{2}{|c|}{ Design A1 } & \multicolumn{2}{|c|}{ Design A2 } & \multicolumn{2}{|l|}{ Design B } \\
\hline & $\begin{array}{l}\text { Matched } \\
\lambda / 2\end{array}$ & $\begin{array}{l}\text { Unmatched } \\
\lambda\end{array}$ & $\begin{array}{l}\text { Matched } \\
\lambda / 2\end{array}$ & $\begin{array}{l}\text { Unmatched } \\
\lambda\end{array}$ & $\begin{array}{l}\text { Matched } \\
\lambda / 2\end{array}$ & $\begin{array}{l}\text { Unmatched } \\
\lambda\end{array}$ \\
\hline Resonance frequency [kHz] & 58 & 68 & 65 & 96 & 57 & 76 \\
\hline Applied Voltage (RMS) [V] & 21 & 70.7 & 7 & 14 & 14 & 24.5 \\
\hline Current (RMS) (max.) [mA] & 302 & 201 & 76 & 310 & 451 & 147 \\
\hline Displacement (max.) $[\mu \mathrm{m}]$ (Peak-to-peak) & 24.1 & $>50$ & 16 & 11 & 22.4 & 37.6 \\
\hline Impedance (min.) $[\Omega]$ & 71 & 352 & 93 & 41 & 31 & 96 \\
\hline Tip velocity (max.) [m/s] & 3 & $>10.5$ & 3.3 & 3.3 & 4 & 8.5 \\
\hline Peak phase angle $\left[{ }^{\circ}\right]$ & 80 & -59 & 47 & 67 & 85 & 70 \\
\hline Phase margin $[\mathrm{Hz}]$ & 1057 & 0 & 234 & 1933 & 2105 & 880 \\
\hline Damping loss [\%] & 9 & 100 & 73.1 & 10 & 10 & 57 \\
\hline Power [W] & 6.3 & 14.2 & 0.5 & 4.9 & 6.3 & 2.1 \\
\hline
\end{tabular}


Fig. 8 Current and phase as a function of frequency for a design $B$ scalpel operating in the unmatched full-wave $(\lambda)$ mode

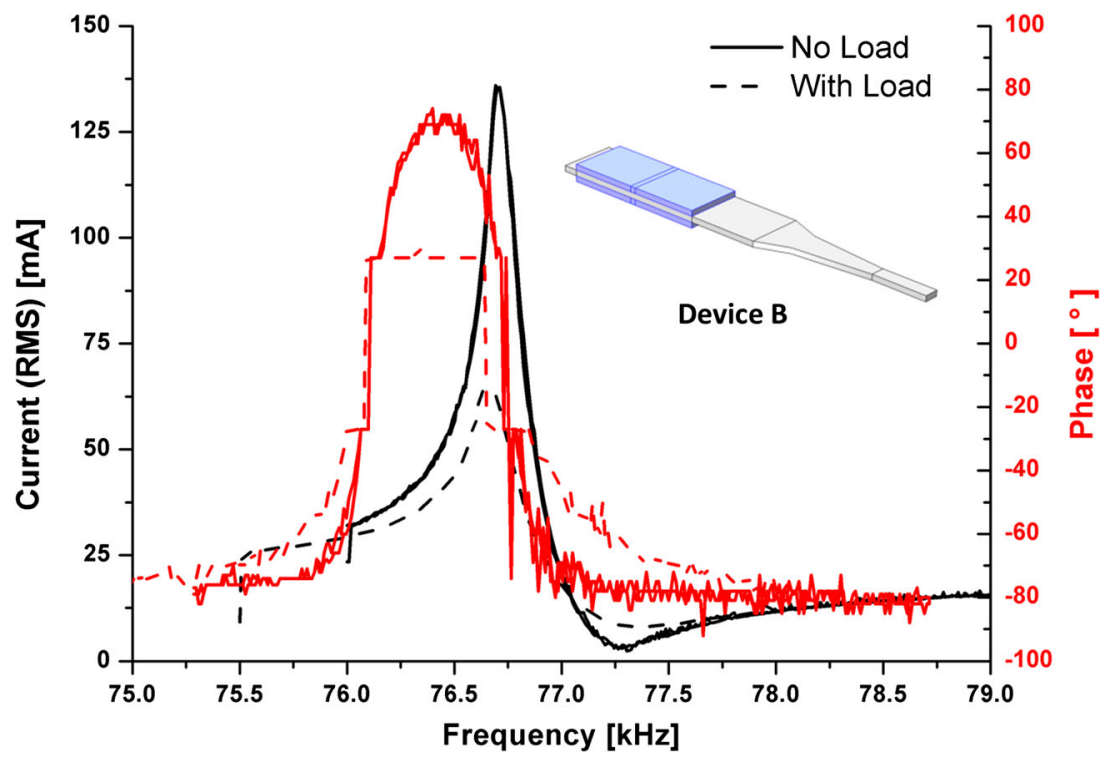

investigated the effects of a.o. application force on the cutting rate of an ultrasonic surgical instrument. They used a 2.2 diameter circular cylinder as blade shape for the end-effector and applied low, average, and high loads of $0.3125,0.625$, and $1.25 \mathrm{~N} / \mathrm{mm}$ for liver tissue and $0.156,0.3125$, and $0.625 \mathrm{~N} / \mathrm{mm}$ for wax, respectively. According to Vaitekunas et al., these forces per $\mathrm{mm}$ cutting depth represent loads applied by a veterinary surgeon cutting a variety of tissues (Vaitekunas et al. 2001).

Based on the results of the finite element modeling, three scalpel designs (A1, A2, and B) were tested as presented in Fig. 7. In two of the designs ( $A 1$ and $B$ ), the resonant frequency of the actuators is matched to the half-wave mode. In the third design (A2), the resonant frequency of the actuators is matched to the full-wave mode.

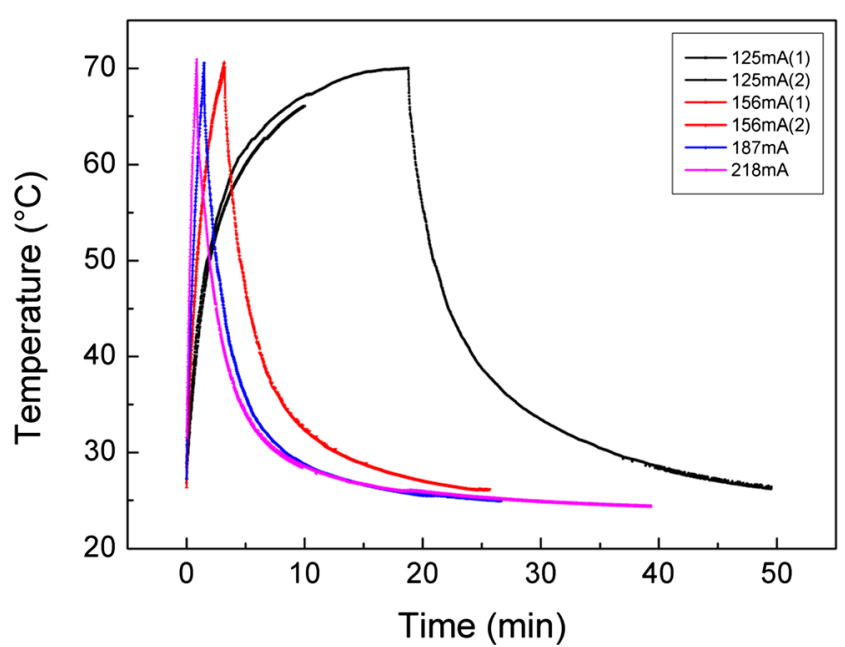

Fig. 9 Temperature increase of scalpel B for various drive currents. At $70{ }^{\circ} \mathrm{C}$ the drive current was removed

\section{Results and discussion}

Design A1, A2 and B scalpels were assembled and characterized. Table 2 presents the comparison of the three scalpel designs which were tested in both the half- and full-wave modes. Scalpels A1 and B were designed such that the resonance of the PZT actuators was matched to the half-wave mode of the Si horn and not matched to the full-wave mode whereas Scalpel A2 was designed such that the resonance of the PZT actuators was matched to the full-wave mode of the $\mathrm{Si}$ horn and not matched to the full-wave mode.

\subsection{Matched resonance}

As expected, transducers in which the resonance mode of the horn is matched to the resonance mode of the PZT actuators were difficult to drive. They had low acoustic gains, and generated more latent heat than acoustic power transferred to a load. The tip displacements and the expected structural gains are found to be lower compared to the unmatched modes (Table 2). This can be explained by the poor mechanical coupling between the PZT and the Si which have very different acoustic impedances. The speed of sound in $\mathrm{Si}$, in the $<110>$ direction, is approximately $9000 \mathrm{~m} / \mathrm{s}$ while the speed of sound in PZT-8 is approximately $3000 \mathrm{~m} / \mathrm{s}$. Therefore, at a given operational frequency, the wavelengths of the two materials do not correspond, thus leading to poor mechanical coupling. Latent heat generation as a result of high currents running through the piezoelectric actuators as well as the poor mechanical coupling between the actuators and the silicon horn cause significant heat generation in the scalpels, resulting in a continuous drift towards lower frequencies when continuously driven in resonance. 
Even though these transducers exhibit wide phase margins, resonance of the piezoelectric material leads to hysteresis which presents a problem for the control electronics lockingin on the resonance frequency of the transducer. A pronounced jump in the phase angle at the resonance frequency causes problems for control algorithms locking onto the zero degree phase crossing. Thus, shifts in the resonance frequency while the load applied to the tip of the transducer is varied causes the system to lose the operational frequency. The main advantage, however, of operating in the matched resonance mode is the transducer's resistance to damping losses which occur when a mechanical load is applied to the tip. Evidence of this is presented in Table 2 comparing all three designs, A1, A2 and B.

In design $\mathrm{A} 2$, the effect of frequency and structure were systematically removed by coupling the natural frequency of the piezoelectric actuators to the full-wave mode. The same stunted displacement amplification, hysteretic behavior and resistance to mechanical damping were observed in all cases where the natural frequencies of the actuators are matched to the natural frequencies of the Si horn.

\subsection{Unmatched resonance}

When the structural resonance of the transducer is designed to be far from the natural frequency of the PZT actuators, the mechanical coupling between the two is found to be much better. The tip displacement of the transducer increases linearly with increasing drive current corresponding to the expected gain of the structure. Small displacements of the PZT actuators (even when the structure is in resonance) alleviates the problem of hysteresis as is apparent from the symmetric frequency response presented in Fig. 8. Thus, while the resonance frequency of scalpels operated in the unmatched resonance mode still shifts due to varying applied mechanical loads and latent heat generation, tracking the operational frequency with

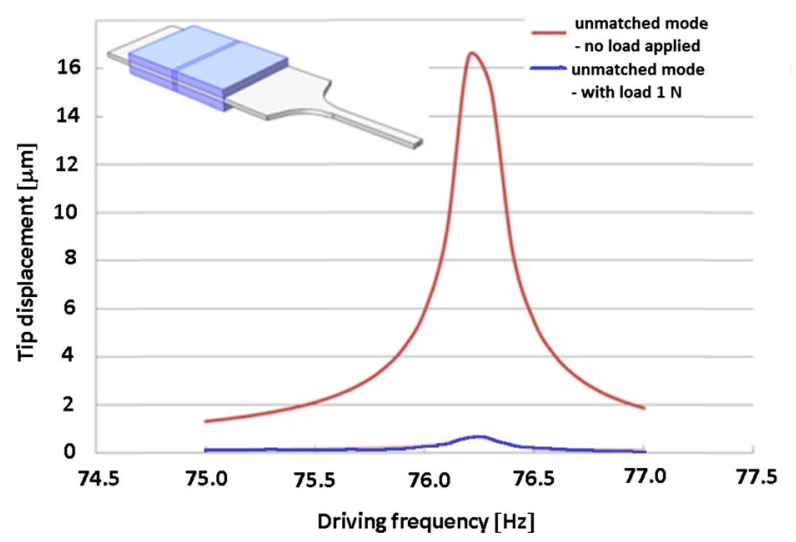

Fig. 10 Influence of a $1 \mathrm{~N}$ load applied to the scalpel tip on its displacement. The amplitude with and without load of an ultrasonic waveguide with geometrical amplification (gain) of 8 (left) compared to waveguide with geometrical amplification (gain) of 4 (right) is shown. control electronics in the unmatched resonance mode is much easier than for the matched resonance design. The mechanical damping, however, resulting from an applied load is much more severe in the unmatched resonance mode of operation as illustrated in Table 2.

\subsection{Geometric amplification}

Design A offers a higher geometrical amplification than design B. Thus, vastly higher displacements are achieved for scalpels of design A when driven in air (i.e., when no load is applied to the tip of the scalpel). However, when a mechanical load is applied to the tip of the scalpel (as it is in cutting applications), design A scalpels with higher geometric amplifications suffer from greater damping as a result of the lever effect. By increasing the geometrical gain, a larger tip displacement is achieved at the cost of a reduced tip force. Experimental results comparing design $\mathrm{A}$ and design $\mathrm{B}$ when driven under static loading conditions confirm the results predicted through finite element modeling (Fig. 10). The impedance of the scalpels with a higher geometrical amplification (design A) increases sharply with the application of a tip load thus requiring much higher voltages to achieve the same tip displacement of the free scalpel. Hence, the rapid impedance fluctuations which occur as a result of a varying mechanical load require large drive voltage swings and extremely fast electronics to precisely control the set peak-to-peak tip displacement of the scalpel. The inability of the control electronics to follow the voltage requirements of the scalpel leads to breakage as a result of driving the scalpels to extreme displacements. Reducing the geometrical amplification of the scalpels (design $\mathrm{B}$ ) reduces the achievable tip displacements, but limits the impedance variations (and therefore operational voltage swings) which increases the integrity of the scalpels.

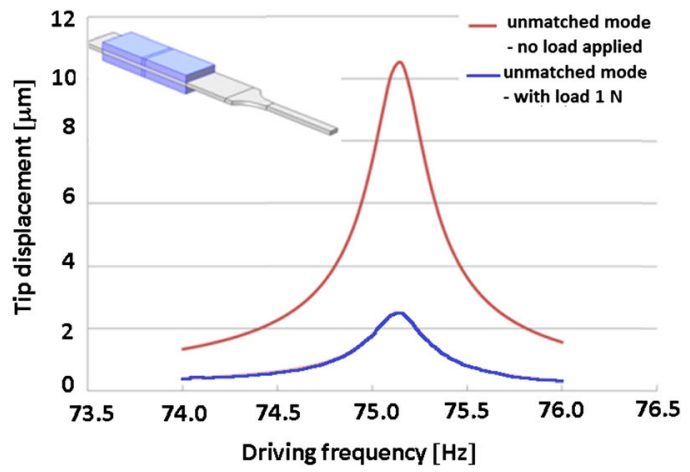

Scalpels with higher geometric amplification show a greater damping of the oscillation amplitude of the tip compared to scalpels with a lower geometric amplification as a result of the lever effect 


\subsection{Thermal stability}

A thermocouple was used to monitor the temperature of the scalpels while in operation. The thermocouple was placed at the back nodal position either directly in contact with the PZT (between the PDMS clamp and PZT surface) or just above the PDMS clamp (approximately $1 \mathrm{~mm}$ from the PZT surface, between the PDMS clamp and the scalpel case). A casing with dimensions (LxWxT) $100 \times 30 \times 15 \mathrm{~mm}^{3}$ was used to hold the scalpel and has been manufactured from ABS using rapid prototyping. The scalpel was supported at the nodes using PDMS spacers with dimensions $10 \times 2 \times 2 \mathrm{~mm}^{3}$. The Silicon scalpel was clamped in between the two shells of the casing using six screws to ensure a uniform pressure on the Si scalpel via the PDMS spacers on both sides of the scalpel. The temperature measurements performed with each of these configurations produced similar results; albeit, the temperature of the second configuration was found to be proportionally lower as expected due to the separation of the thermocouple and the surface of the PZT. The scalpels were driven to a maximum temperature of $70{ }^{\circ} \mathrm{C}$ (thermocouple in direct contact with the PZT). The temperature of the scalpels as a function of time was recorded for several input amplitudes $\left(\mathrm{I}_{\max }=250 \mathrm{~mA}\right)$. Upon reaching $70^{\circ} \mathrm{C}$, the scalpels were then allowed to cool back to ambient temperature. As shown in Fig. 9, the time needed to reach $70{ }^{\circ} \mathrm{C}$ was reduced for higher drive levels. At an input current of $I=125 \mathrm{~mA}$, the scalpel takes $18 \mathrm{~min}$ to reach the set limit of $70^{\circ} \mathrm{C}$ while at current of $I=188 \mathrm{~mA}$, the time required is only $40 \mathrm{~s}$. Cooling tests were also performed by introducing an air flow into the casing holding the scalpel. A small, flexible tube connected to the air supply of the building was inserted into the opening used for the electrical connections. Using a constant flow of air into the chamber, the temperature of the scalpels could be stabilized below $70^{\circ} \mathrm{C}$ for longer driving periods.

\subsection{In-vitro dissection}

Operational cutting tests were performed in the lab in water as well as on tangerines, squash balls, leather, chicken and pork tissue. Temperature increases during operation which caused the resonant frequency of the scalpel to drop during operation, but remained in resonance using fast tracking electronics. Images of the performed cutting tests are shown in Fig. 11.

Cutting of chicken skin has been performed by hand using a cutting speed of approximately $4 \mathrm{~mm} / \mathrm{s}$ with an initial depth of 1-2 mm. As soon as the skin has been completely cut, the cutting speed has been increased to about $6-8 \mathrm{~mm} / \mathrm{s}$ with a cutting depth of 4-5 mm as muscle tissue is much easier cut. Coagulation of the tissue can be observed at the border of the cut where white lines of coagulated tissue can be discerned on both sides of the cut having a width of about $0.4-1 \mathrm{~mm}$ (Fig. 11 right). As can be seen on Fig. 11 left, the coagulation
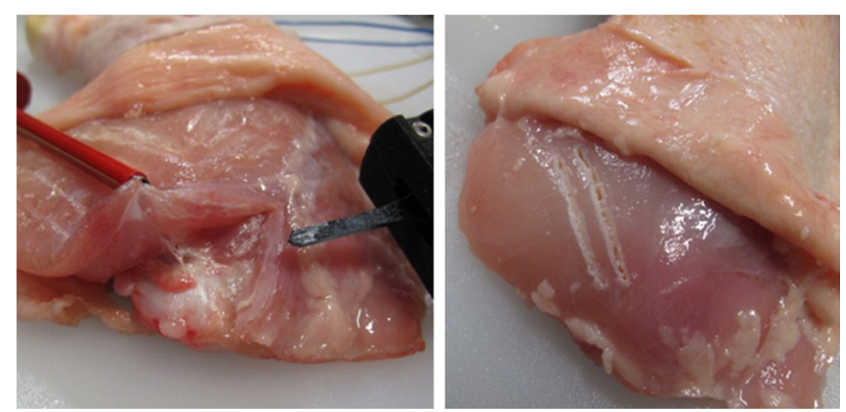

Fig. 11 Dissection test with Si scalpel B on a chicken leg. Left: the tissue surface inside a cut is exposed showing less coagulation compared to the top surface of tissue (right) after cutting with the ultrasonic microscalpel where coagulation can be observed as white lines at the border of the cut

is much less pronounced at the tissue inside the cut. This corresponds well with the observations made by Ebina et al. who have shown that tissue cut with an ultrasonic instrument having a vibrating tip at a speed of $2 \mathrm{~m} / \mathrm{s}$ or less is coagulated by the longitudinal vibration but not by the lateral vibration (Ebina et al. 2007). As the vibration speed of the tip used in Fig. 11 was $1.7 \mathrm{~m} / \mathrm{s}$, the coagulation effect of the surface can be explained by the initial contact of the tip with the tissue surface when the first cut line was drawn and the tissue was subject to longitudinal vibration. Inside a cut, the tissue is exposed to the side surface of the scalpel tip where the lateral vibrations of the scalpel cause shear strain in the tissue and less heat compared to longitudinal vibrations. More detailed experiments with defined cutting load and speed will be performed and reported in an upcoming publication.

\section{Conclusions}

We have presented a novel silicon micromachined ultrasonic scalpel which is composed of a flat silicon micromachined acoustic horn and two PZT actuators bonded to either face of the Si horn which is small, light and powerful and is ideally suited for high precision surgery.

Important design parameters for efficient and robust $\mathrm{Si}$ based microscalpels have been identified. The microscalpels were manufactured cost-effectively by microfabrication batch processing. A final thermal oxidation step has been included to improve the biocompatibility and reliability of the Si. Impedance analysis, displacement measurement via LDV and cutting tests allowed characterization of the fabricated devices. The optimized micro-cutter has been successfully used to cut chicken tissue and was able to deliver sufficient power in order to dissect and coagulate the tissue. Through optimization, we have brought the Si microscalpel one step closer to compact and ergonomic ready-to-use applications in surgery.

Acknowledgments This research was supported by the Swiss Federation's Commission for Technology and Innovation (grant CTI \# 11309.1 PFLS-LS. 


\section{References}

S. Albert, C. Guedon, C. Halimi, J.P. Cristofari, B. Barry, The Use of Harmonic Scalpel for Free Flap Dissection in Head and Neck Reconstructive Surgery. Plast. Surg. Int. (2012). doi:10.1155/2012/ 302921

J.F. Amaral, Ultrasonic dissection. Endosc. Surg. Allied. Technol. 2, 181185 (1994)

D.N. Armstrong, W.L. Ambroze, M.E. Schertzer, G.R. Orangio, Harmonic Scalpel vs. electrocautery hemorrhoidectomy: a prospective evaluation. Dis. Colon Rectum 44, 558-564 (2001)

S.A. Boddy, J.W. Ramsay, S.S. Carter, P.J. Webster, D.A. Levison, H.N. Whitfield, Tissue effects of an ultrasonic scalpel for clinical surgical use. Urol. Res. 15, 49-52 (1987)

L.J. Bond, W.W. Cimino, Physics of ultrasonic surgery using tissue fragmentation: Part II. Ultrasound Med. Biol. 22, 101-117 (1996a)

L.J. Bond, W.W. Cimino, Physics of ultrasonic surgery using tissue fragmentation. Ultrasonics 34, 579-585 (1996b)

P.S. Brazio, P.C. Laird, C. Xu, J. Gu, N.S. Burris, E.N. Brown, Z.N. Kon, R.S. Poston, Harmonic scalpel versus electrocautery for harvest of radial artery conduits: reduced risk of spasm and intimal injury on optical coherence tomography. J. Thorac. Cardiovasc. Surg. 136, 1302-1308 (2008). doi:10.1016/j.jtcvs.2008.05.060

T.E. Burdette, C.L. Kerrigan, K. Homa, K.A. Homa, Harmonic scalpel versus electrocautery in breast reduction surgery: a randomized controlled trial. Plast. Reconstr. Surg. 128, 243e-249e (2011). doi:10. 1097/PRS.0b013e318221da3e

M. Cikirikcioglu, M. Yasa, Z. Kerry, H. Posacioglu, M. Boga, T. Yagdi, N. Topcuoglu, S. Buket, A. Hamulu, The effects of the Harmonic Scalpel on the vasoreactivity and endothelial integrity of the radial artery: a comparison of two different techniques. J. Thorac. Cardiovasc. Surg. 122, 624 (2001)

W.W. Cimino, L.J. Bond, Physics of ultrasonic surgery using tissue fragmentation: Part I. Ultrasound Med. Biol. 22, 89-100 (1996)

K. Ebina, H. Hasegawa, H. Kanai, Investigation of frequency characteristics in cutting of soft tissue using prototype ultrasonic knives. Jpn. J. Appl. Phys. 46, 4793-4800 (2007)

E. Eisner, The design of resonant vibrators, in: physical acoustics principles and methods (Academic Press Inc, New York, 1964), pp. 353-363

F. Ericson, J.-Å. Schweitz, Micromechanical fracture strength of silicon. J. Appl. Phys. 68, 5840-5844 (1990). doi:10.1063/1.346957

C. Funke, S. Wolf, D. Stoyan, Modeling the tensile strength and crack length of wire-sawn silicon wafers. J. Sol. Energy Eng. 131, 011012-011012 (2009). doi:10.1115/1.3028048

M. Kauko, New techniques using the ultrasonic scalpel in laparoscopic hysterectomy. Curr. Opin. Obstet. Gynecol. 10, 303-305 (1998)

C.D. Kelman, Phaco-emulsification and aspiration. A new technique of cataract removal. A preliminary report. Am. J. Ophthalmol. 64, 23 35 (1967)

B. Kiaii, W.D. Boyd, R. Rayman, W.B. Dobkowski, S. Ganapathy, G. Jablonsky, R.J. Novick, Robot-assisted computer enhanced closedchest coronary surgery: preliminary experience using a Harmonic Scalpel and ZEUS. Heart Surg. Forum 3, 194-197 (2000)

Y. Kuang, M. Sadiq, S. Cochran, Zhihong Huang, Effects of power levels and soft tissue loads on an ultrasonic planar tool driven by PMN-PT d31 plates. Ultrason. Symp. IUS. IEEE. Int. 1432-1435 (2013). doi: 10.1109/ULTSYM.2013.0363

A. Lal, Micromachined silicon ultrasonic longitudinal mode actuators: theory and applications to surgery, pumping and atomization (University of California, Berkeley, 1996)

A. Lal, R.M. White, Silicon microfabricated horns for power ultrasonics. Sens. Actuators Phys 54, 542-546 (1996). doi:10.1016/S09244247(97)80011-8
P. Lamm, G. Juchem, P. Weyrich, A. Schütz, B. Reichart, The harmonic scalpel: optimizing the quality of mammary artery bypass grafts. Ann. Thorac. Surg. 69, 1833-1835 (2000)

F. Lumachi, A.A. Brandes, P. Burelli, S.M.M. Basso, M. Iacobone, M. Ermani, Seroma prevention following axillary dissection in patients with breast cancer by using ultrasound scissors: a prospective clinical study. Eur. J. Surg. Oncol. J. Eur. Soc. Surg. Oncol. Br. Assoc. Surg. Oncol. 30, 526-530 (2004). doi:10.1016/j.ejso.2004.03.003

A.L. Melck, S.M. Wiseman, Harmonic scalpel compared to conventional hemostasis in thyroid surgery: a meta-analysis of randomized clinical trials. Int. J. Surg. Oncol. (2010). doi:10.1155/2010/396079

F.U. Metternich, C. Sagowski, S. Wenzel, T. Jäkel, R. Leuwer, U. Koch, Preliminary results for superficial parotidectomy using the ultrasonically activated scalpel (Ultracision Harmonic Scalpel). Laryngorhinootologie 82, 514-519 (2003). doi:10.1055/s-200340894

T. Nagoya, M.K. Kurosawa, A micro ultrasonic scalpel with sensing function. IEEE. 1070-1073 (2003). doi:10.1109/ULTSYM.2003. 1293585

B.J. O’Daly, E. Morris, G.P. Gavin, J.M. O’Byrne, G.B. McGuinness, High-power low-frequency ultrasound: a review of tissue dissection and ablation in medicine and surgery. J. Mater. Process. Technol. 200, 38-58 (2008). doi:10.1016/j.jmatprotec.2007.11.041

G. Pavlíková, R. Foltán, M. Horká, T. Hanzelka, H. Borunská, J. Šedý, Piezosurgery in oral and maxillofacial surgery. Int. J. Oral Maxillofac. Surg. 40, 451-457 (2011). doi:10.1016/j.ijom.2010.11. 013

K.E. Petersen, Silicon as a mechanical material. IEEE Proc. 70, 420-457 (1982)

M.R. Sadiq, Y. Kuang, Z. Huang, S. Cochran, Ultrasonic cutting with a d31-mode PMN-PT-driven planar tool. Ultrason. Symp. IUS. IEEE. Int. 2189-2192 (2011). doi:10.1109/ULTSYM.2011.0543

I. Sasagawa, H. Suzuki, T. Izumi, Y. Suzuki, T. Tateno, T. Nakada, Posterior retroperitoneoscopic partial adrenalectomy using ultrasonic scalpel for aldosterone-producing adenoma. J. Endourol. Endourol. Soc. 14, 573-576 (2000). doi:10.1089/ 08927790050152177

J.A. Sherman, H.T. Davies, Ultracision ${ }^{\circledR}$ : the harmonic scalpel and its possible uses in maxillofacial surgery. Br. J. Oral Maxillofac. Surg. 38, 530-532 (2000). doi:10.1054/bjom.2000.0502

A. Stoff, M.A. Reichenberger, D.F. Richter, Comparing the ultrasonically activated scalpel (Harmonic) with high-frequency electrocautery for postoperative serous drainage in massive weight loss surgery. Plast. Reconstr. Surg. 120, 1092-1093 (2007). doi:10.1097/01.prs. 0000278224.14221.c9

S. Takao, H. Shinchi, K. Maemura, T. Aikou, Ultrasonically activated scalpel is an effective tool for cutting the pancreas in biliarypancreatic surgery: experimental and clinical studies. J. Hepatobiliary Pancreat. Surg. 7, 58-62 (2000). doi:10.1007/ s005340000070058.534

J.J. Vaitekunas, F.B. Stulen, E.S. Grood, Effects of frequency on the cutting ability of an ultrasonic surgical instrument. 31st Annu. Ultrason. Ind. Assoc. Symp. Atlanta USA. (2001)

R.A. Walker, Z.A. Syed, Harmonic scalpel tonsillectomy versus electrocautery tonsillectomy: a comparative pilot study. Otolaryngol.Head Neck Surg. Off. J. Am. Acad. Otolaryngol. Head Neck Surg. 125, 449-455 (2001)

D.-A. Wang, W.-Y. Chuang, K. Hsu, H.-T. Pham, Design of a Bézierprofile horn for high displacement amplification. Ultrasonics 51, 148-156 (2011). doi:10.1016/j.ultras.2010.07.004

S. Watanabe, H. Sato, K. Tawaraya, M. Tsubota, M. Endo, M. Seki, Advantages and disadvantages of harmonic scalpel in thoracic surgery. Kyobu Geka 51, 374-378 (1998) 